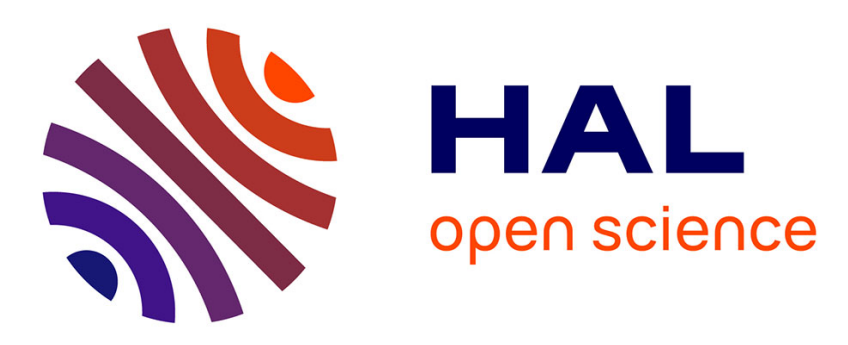

\title{
A novel method using EEG to characterize the cortical processes involved in active and passive touch
}

Athanasia Moungou, Eric Vezzoli, Celine Lombart, Betty Lemaire-Semail, Jean-Luis Thonnard, André Mouraux

\section{- To cite this version:}

Athanasia Moungou, Eric Vezzoli, Celine Lombart, Betty Lemaire-Semail, Jean-Luis Thonnard, et al. A novel method using EEG to characterize the cortical processes involved in active and passive touch. IEEE - Haptic Symposium 2016, Apr 2016, Philadelphia, United States. 10.1109/HAPTICS.2016.7463178 . hal-01318133

\section{HAL Id: hal-01318133 \\ https://inria.hal.science/hal-01318133}

Submitted on 20 May 2016

HAL is a multi-disciplinary open access archive for the deposit and dissemination of scientific research documents, whether they are published or not. The documents may come from teaching and research institutions in France or abroad, or from public or private research centers.
L'archive ouverte pluridisciplinaire HAL, est destinée au dépôt et à la diffusion de documents scientifiques de niveau recherche, publiés ou non, émanant des établissements d'enseignement et de recherche français ou étrangers, des laboratoires publics ou privés. 


\title{
A novel method using EEG to characterize the cortical processes involved in active and passive touch *
}

\author{
A. Moungou, E. Vezzoli, C. Lombart, B. Lemaire-Semail, J.-L. Thonnard, A. Mouraux
}

\begin{abstract}
We present a novel method to compare brain responses to identical tactile stimuli in active and passive touch. Using electroencephalography (EEG) to record steady-state evoked brain potentials (SS-EPs), our goal was to characterize the cortical activity related to the tactile exploration of a textured surface. For this purpose, we used a novel tactile display, which is able to produce tactile texture experiences using ultrasonic stationary waves to transiently modulate tangential friction between the finger and the display. Because the change in friction depends on the amplitude of the ultrasonic vibrations, modulation of ultrasonic vibration amplitude was used to periodically modulate friction at a frequency of $11 \mathrm{~Hz}$, producing a tactile percept resembling that of a square-wave grating. After recording the exact motion of the fingertip and normal force used for each trial while one participant freely explored the surface (active touch condition), the same motion and normal force was reproduced by a highprecision robotic device with force feedback (passive touch condition). Frequency analysis of the recorded EEG signals showed that, for both active and passive touch conditions, the interactions between the fingertip and the plate elicited a robust SS-EP at $11 \mathrm{~Hz}$, corresponding to the frequency of friction modulation, maximal over the parietal region contralateral to the stimulated finger. Our results suggest that the cortical activity related to active and passive touch can be characterized in humans by combining the recording of SS-EPs with an ultrasonic device generating a periodic tactile experience whose frequency is independent of finger movements.
\end{abstract}

\section{INTRODUCTION}

Over the past decades, there has been an increased interest in studying touch perception using functional neuroimaging techniques [1-3]. The notion that active and passive tactile exploration could involve distinct processes was first proposed by Gibson [4]. It has since been suggested that when people perform voluntary finger movements (active touch), different neural mechanisms are recruited as compared to when the same movement is produced externally (passive touch) $[5,6]$.

Touch is essentially a dynamic process and movement is considered to be a decisive factor for tactile perception in general. Stimuli can be dynamic or static, i.e. moving or

*This work was funded by the FP7 Marie Curie Initial Training Network A. Moungou, C. Lombart, J.-L. Thonnard, and A. Mouraux are with the Institute of Neuroscience (IoNS), Université catholique de Louvain, Brussels, Belgium 1200 (phone: +3227645447; e-mail: athanasia.moungou@uclouvain.be, cecile.lombart@uclouvain.be, jeanlouis.thonnard@uclouvain.be, andre.mouraux@uclouvain.be).

E. Vezzoli and B. Lemaire-Semail are with L2EP-IRCICA, University of Lille, Lille, France 59650 (phone: +33613115733; e-mail: eric.vezzoli@ed.univ-lille1.fr, betty.semail@polytech-lille.fr). stationary with respect to the skin. It is widely accepted in the literature that tactile perception is better with dynamic stimuli. For instance, when we move our finger along a surface, we are able to discriminate even slight differences compared to when the finger stays static [4, 7-12]. When dynamic stimuli are used, both slowly and rapidly adapting mechanoreceptors contribute to the tactile experience. In contrast, when the tactile stimulus is static, only slowly adapting mechanoreceptors are engaged.

On the other hand, it is also known that movement reduces the excitability of the primary somatosensory cortex to tactile inputs, an effect widely referred to as movementrelated gating of sensory transmission [5, 13, 14], where both cutaneous and proprioceptive inputs are gated/suppressed [15-18]. This effect has been reported to take place at the dorsal column nuclei of the lemniscal pathway [15].

There are contradictory results in the literature when active touch is compared to passive touch, and these results could be dependent on the nature of the task performed by the participants. Even if active touch has the advantage of providing a more natural feeling of exploration, studies have suggested that somatosensory input generated during passive touch elicits responses of greater magnitude in the primary somatosensory cortex as compared to active touch $[5,19]$.

Until recently, most studies have focused on the perception of textured stimuli (such as Braille dot patterns, surfaces of varying roughness) in active and passive touch using roughness discrimination tasks $[2,6,11,20]$. Interestingly, in a recent study investigating the neural mechanisms underlying active and passive touch, using a roughness categorization task and fMRI, greater activation was found for active touch [21]. Still, it is not fully understood how active or passive touch influences the processing of tactile information in the somatosensory cortices.

Passively imposed movements can never exactly reproduce the pattern of afferent feedback associated with voluntary movement, especially in relation to feedback from muscle afferents and joint receptors since their sensitivity is modulated by fusimotor and/or skeletomotor activity. Unlike passive touch, active touch involves an input from proprioception [22]. Using, thus, identical stimuli in both active and passive touch is technically challenging.

The aim of the present study was to test a novel approach to investigate the brain responses under identical exploratory conditions in active and passive touch conditions using electroencephalography (EEG) to record steady-state evoked potentials (SS-EPs). 
SS-EPs are induced by the periodic modulation of a stimulus feature. For instance, periodically modulating the amplitude of a mechanical stimulus at a given frequency elicits a consistent peak in the EEG frequency spectrum at the frequency of modulation and its harmonics. This response is thought to result from synchronized periodic activity generated in neuronal populations responding to the periodically-modulated feature [23]. In the somatosensory modality, several studies have used SS-EPs to characterize the cortical activity related to the perception of pure mechanical vibrations [24-26] or electrical stimulation [27, 28]. The majority of these studies have focused on frequencies around $20 \mathrm{~Hz}$.

In most of the studies employing the SS-EPs approach, the index finger is at rest while vibratory and mechanical stimuli are applied on the index. Consequently, obtaining brain responses during the active exploration of a surface, regardless of the movement, is a first challenge that needs to be considered.

For the purposes of this study, a novel tactile display was used to modulate the tangential friction between the fingertip and the display. Two different methods are currently available to modulate the tangential force of a finger sliding on a flat surface: electrovibration and ultrasonic vibrations. The first increases the friction due to electrostatic attraction of the fingertip [29, 30]. The second reduces friction as a function of ultrasonic vibration amplitude [31-33]. The display used in the present study is based on the generation of ultrasonic vibrations [34]. Since the change in friction depends on the amplitude of the ultrasonic vibrations, modulation of vibration amplitude was used to periodically modulate friction at a frequency of $11 \mathrm{~Hz}$, producing a tactile percept resembling that of a square-wave grating [35].

Our goal was to record SS-EPs and compare the brain activity at the frequency of friction modulation in active and passive touch. For this purpose, we first recorded SS-EPs while one participant explored freely the display (active touch condition). During these trials, the exact motion of the fingertip and normal force applied by the fingertip against the display were recorded such as to reproduce the exact same motion and force using a high-precision robotic device with force feedback control (passive touch condition).

\section{MATERIALS AND METHODS}

\section{A. Stimulus - Tactile Display}

A novel tactile display was used, able to produce localized tactile feedback and texture rendering to the fingertip using ultrasonic stationary waves to transiently modulate tangential friction between the finger and the display. The brain responses obtained during active exploration of the display were compared to those obtained during passive exploration of the display.

The display consisted of a 70x40 mm aluminum plate. Ultrasonic vibrations of the plate were used to reduce the coefficient of friction between the surface and the fingertip ( $25 \mathrm{kHz}$, maximum amplitude: $2.5 \mu \mathrm{m}$ ). Because the change in friction depends on the amplitude of the ultrasonic vibrations, modulation of vibration amplitude was used to periodically modulate friction at a frequency of $11 \mathrm{~Hz}$. This produced a tactile sensation of a square-wave grating.

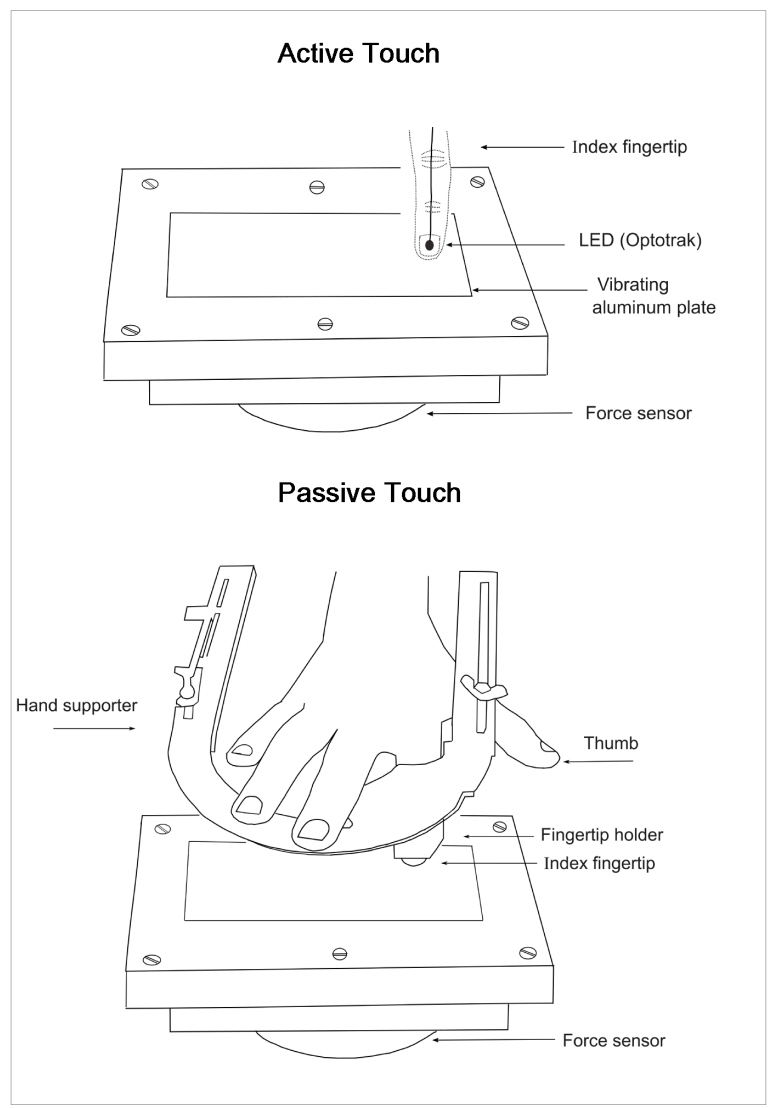

Figure 1. Schematic view of the experimental setup in active and passive touch. In active touch, the LED sensor recording the fingertip's movement is glued on the nail, while the force is recorded. In passive touch the right index finger is maintained by a hand support and a fingertip holder.

\section{B. Apparatus}

A schematic view of the experimental setup is shown in Fig. 1.

In the active touch condition, the position of the subject's finger was measured by means of an infrared optical tracking system and an infrared LED marker bonded to the nail (Optotrak, Northern Digital Inc., Waterloo, Ontario, Canada). This motion-tracking device collects data with a sampling rate of $50 \mathrm{~Hz}$ and a mean accuracy of $1 \mathrm{~mm}$ for the displacement. Furthermore, a forces and torque transducer (Nano40 F/T transducer, ATI Industrial Automation, USA) was mounted under the aluminum plate to record the normal and tangential forces during the active tactile exploration of the plate. This transducer is able to record forces with a resolution of $0.004 \mathrm{~N}$ in each direction (along the $\mathrm{x}, \mathrm{y}, \mathrm{z}$ axes).

In the passive touch condition, the fingertip was maintained at a fixed position using a retainer (Fig. 1) and the movements of the plate relative to the fingertip, as well as the normal force between the plate and fingertip were reproduced using a four-axis robot (four-axis SCARA HS series 4535G. DENSO, USA), driven by the LabVIEW 
(National Instruments, USA) software. This robotic device has a precision accuracy of $15 \mu \mathrm{m}$ for the displacements. The forces and torque transducer under the plate were used to record the normal and tangential forces during the passive touch condition, such as to ensure that the stimulation of the fingertip during active and passive touch conditions were similar (Fig. 2).

The experiment consisted of a total of two blocks: one block of 30 trials during which the participant performed active back and forth movements of the fingertip against the plate (each lasting 20 seconds) and one block of 30 trials during which the motion and normal force were reproduced in the passive touch condition. There was a random pause of 4-6 $\mathrm{s}$ between the strokes in each block.

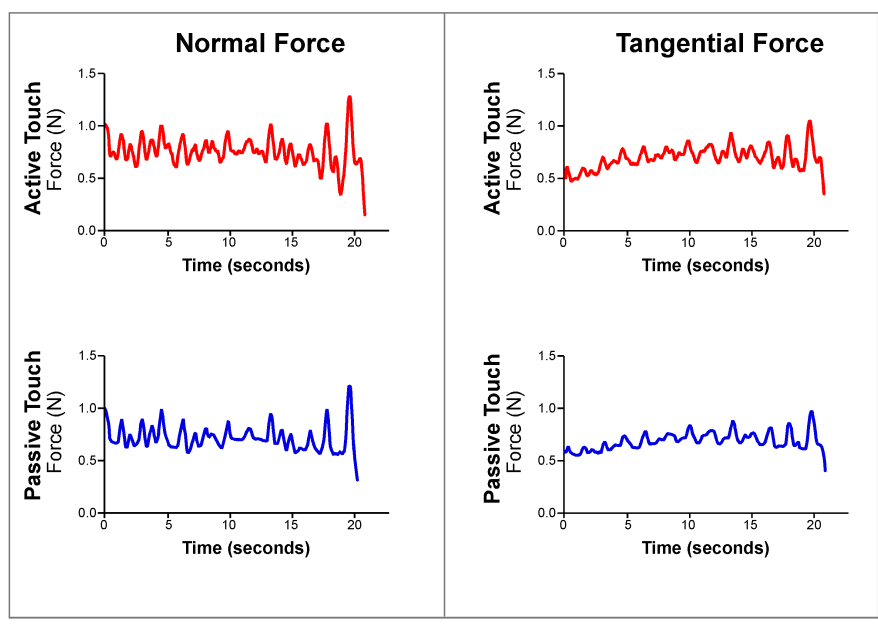

Figure 2. Normal and tangential forces were measured using a forces and torque transducer. The graphs show the similarity of the forces for one trial in both conditions. (x-axis: time, in seconds; y-axis: force, in Newton). Each trial lasted $20 \mathrm{~s}$.

\section{Data acquisition}

EEG data were collected from one healthy volunteer (aged 25 years old, female). The participant was seated comfortably in front of the apparatus. Written informed consent was obtained. The study was approved by the local Ethics Committee and conformed to the latest revision of the Declaration of Helsinki.

\section{Electrophysiological measurements}

EEG signals were recorded using $64 \mathrm{Ag}-\mathrm{AgCl}$ electrodes placed on the scalp according to the International 10/10 system (Waveguard64 cap, Cephalon A/S, Denmark). Electrode impedances were kept below $5 \mathrm{k} \Omega$. Signals were amplified and digitized using a sampling rate of $1000 \mathrm{~Hz}$, using an average reference (64-channel high-speed amplifier, Advanced Neuro Technology, The Netherlands). During the recording, the participant was instructed to attend a fixation cross and listened to white noise presented through headphones to avoid any contribution of auditory input to the recorded EEG responses. A trigger was sent to the EEG system at the onset of each motion.

\section{EEG data analysis}

The EEG data were analyzed in MATLAB (The Mathworks, MA) using the Letswave6 toolbox (http:/www.nocions.org/letswave; see also [36]). Statistical analyses were performed using SPSS 22 (IBM, USA). A notch filter was applied to remove environmental noise. The data were imported and high-pass filtered at $0.1 \mathrm{~Hz}$ to remove slow drifts in the recorded signals. EEG epochs were segmented from $0 \mathrm{~s}$ to $+20 \mathrm{~s}$ relative to the onset of each motion for both active and passive touch conditions. These EEG segments corresponded to the time interval during which the finger moved along the device.

Artifacts due to eye blinks or eye movements were removed using an independent component analysis (FastICA algorithm) for blind-source decomposition of the data into 64 components [37]. The data were re-referenced offline to an average reference. The obtained signals were then transformed in the frequency domain using a discrete Fourier Transform [38], yielding a frequency spectrum ranging from 0 to $500 \mathrm{~Hz}$. The frequency resolution of the FFT was $0.05 \mathrm{~Hz}$. Amplitude spectra were then obtained using the modulus of the complex Fourier coefficients (Fig. 3).

Assuming additive noise, the obtained EEG frequency spectra may be expected to correspond to the sum of (1) EEG activity induced by the somatosensory stimulation, i.e. somatosensory SS-EPs and (2) unrelated background noise due, for instance, to spontaneous EEG activity, muscle activity or eye movements. Hence, to obtain estimates of the magnitude of the elicited SS-EPs, the contribution of this noise was removed by subtracting, at each bin of the frequency spectra, the average amplitude measured at surrounding frequency bins (three frequency bins ranging from -2 to -5 bins and three frequency bins ranging from +2 to +5 bins). This procedure is justified by the fact that, in the absence of an SS-EP, the amplitude at a given frequency should be similar to the amplitude of the mean of the surrounding frequencies $[36,39,40]$. Thus, in the absence of an SS-EP, the noise-subtracted amplitude should tend towards zero. As a final step, all EEG epochs were averaged across trials in the time domain.

\section{Normal and tangential force analysis}

In order to assess the changes in normal force and tangential force induced by the sliding of the fingertip against the plate in both conditions, the measures obtained from the forces and torque transducer were analyzed in the frequency domain using the same procedures used to analyze the EEG data.

\section{Statistical analyses}

To assess the significance of the elicited responses, the maximal amplitude from the noise-subtracted spectra of EEG signals measured at central and parietal electrodes, contralateral to the stimulated fingertip, was calculated (i.e. T7, C1, C3, C5, CP1, CP3, CP5, P1, P3, P5, P7, TP7) across trials (i.e. 30). Considering their position relative to the left primary somatosensory cortex, these electrodes may be 
expected to elicit the strongest response to tactile stimulation of the right index fingertip [e.g. [41]].

One-sample t-tests against zero were used to assess whether the amplitude of the signal obtained at the expected SS-EP frequency $(11 \mathrm{~Hz})$ was significantly greater than zero.

Significance level was set at $\mathrm{p}<0.05$.

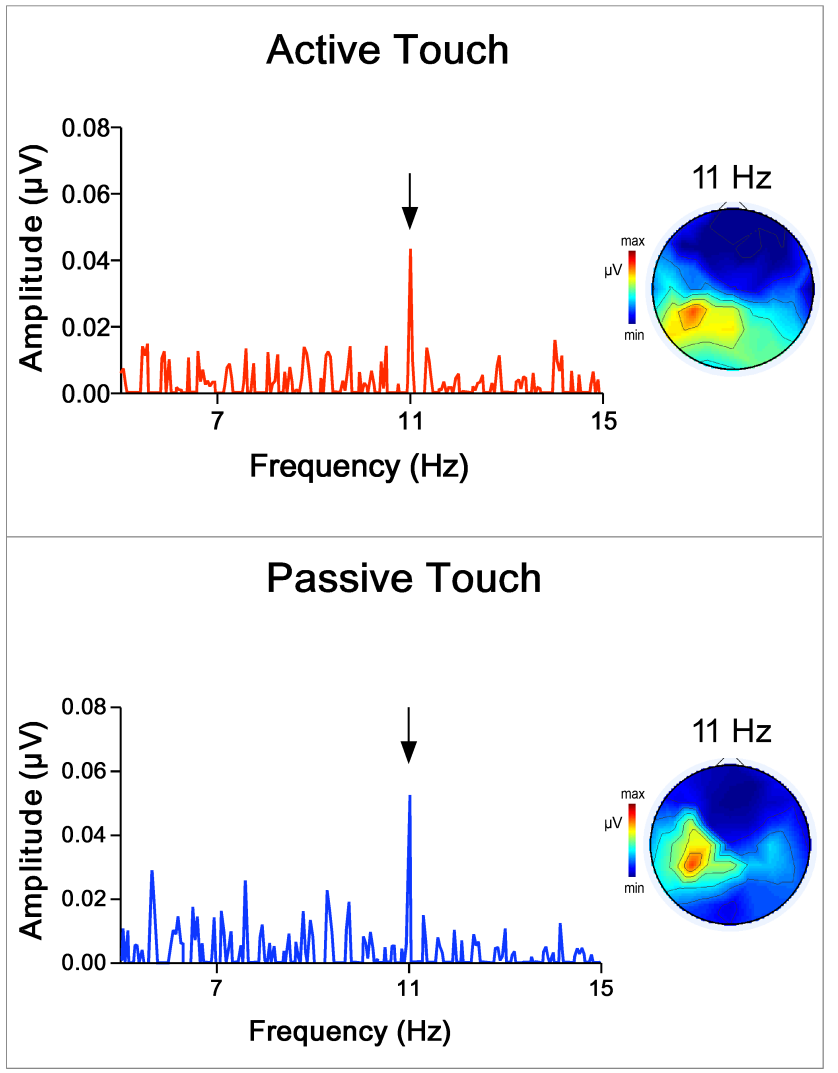

Figure 3. Grand-average EEG frequency spectra across trials during active (above) and passive (below) exploration of the right index finger tip across the display. The vertical arrows mark the frequency of the expected SSEPs (first harmonic). $x$-axis: frequency (Hz), $y$-axis: average noisesubtracted amplitude $(\mu \mathrm{V})$ of the EEG signals recorded from left parietal electrodes. The scalp topographies show the topographical distribution of the signal at the expected SS-EP frequency.

\section{RESULTS}

\section{Active Touch condition}

In the active touch condition, a significant increase of EEG signal amplitude was observed at $11 \mathrm{~Hz}$, corresponding to the expected frequency of friction modulation elicited by the interaction of the fingertip with the tactile display (Fig. 3 ). The scalp topography of the elicited response was maximal over the left parietal region contralateral to the stimulated fingertip. The one sample t-test showed that the amplitude at $11 \mathrm{~Hz}$ was significantly greater than zero $(\mathrm{t}(29)$ $=6.750, \mathrm{p}<0.001,95 \%$ CI [0.027, 0.051]).

\section{Passive Touch condition}

In the passive touch condition, a robust SS-EP was also elicited at $11 \mathrm{~Hz}$ (Fig. 3), $(\mathrm{t}(29)=7.299, \mathrm{p}<0.001,95 \% \mathrm{CI}$ $[0.041,0.073])$. Like in active touch condition, the scalp topography of this SS-EP was clearly maximal over the left parietal region.

The paired-samples t-test comparing the amplitude of SSEPs for both conditions revealed a significant difference $(\mathrm{t}(29)=-2.110, \mathrm{p}=0.044, \mathrm{~d}=0.477,95 \%$ CI [-0.034, $0.001])$.

\section{Force Data Analysis}

As expected, the $11 \mathrm{~Hz}$ friction modulation of the plate against the fingertip induced a periodic variation of the tangential force (active touch: $\mathrm{t}(29)=8.952, \mathrm{p}<0.001,95 \%$ CI [0.023, 0.028]; passive touch: $\mathrm{t}(29)=7.890, \mathrm{p}<0.001$, $95 \%$ CI $[0.017,0.030])$. A significant periodic modulation of the normal force was also observed (active touch: $\mathrm{t}(29)=$ 7.627, $\mathrm{p}<0.001,95 \%$ CI [0.002, 0.004], passive touch: $\mathrm{t}(29)$ $=9.939, \mathrm{p}<0.001,95 \%$ CI $[0.004,0.006]$. The paired samples t-tests did not reveal any significant difference between the modulation of the normal and tangential forces during active and passive touch conditions (normal force: $(\mathrm{t}(29)=-4.576 ., \mathrm{p}=0.074,95 \%$ CI [-0.041, 0.002], tangential force: $\mathrm{t}(29)=-2.888, \mathrm{p}=0.222,95 \%$ CI $[-0.058,0.016]$.

These results indicate that the tactile input generated in the two conditions was not significantly different.

In summary, friction modulation induced by active and passive exploration of the tactile display elicited significant SS-EPs with comparable scalp topographies in both active touch and passive touch conditions.

\section{DISCUSSION}

The aim of this experiment was to isolate and characterize the cortical processing of vibrotactile input generated by the interactions between the fingertip and a textured surface in active vs. passive touch conditions. For this purpose, an ultrasonic tactile display was used to generate a periodic 11 $\mathrm{Hz}$ modulation of friction independent of the fingertip movement against the display. Furthermore, a force feedback-controlled robot was used to reproduce, in the passive touch condition, the exact movements and normal force used during active touch.

So far, the difficulty to match fingertip stimulation in active and passive touch conditions has been the main limitation to allow comparing the brain responses elicited in the two conditions.

By periodically modulating the coefficient of friction at a constant frequency of $11 \mathrm{~Hz}$, we expected that scanning of the fingertip against the display would induce an SS-EP at the corresponding frequency, tagging the cortical activity related to the processing of this tactile input, both when exploring the display voluntarily (active touch) and when the same movements were reproduced by the robot (passive touch). Analysis of the actual normal and tangential forces confirmed that the tactile stimulation was matched in the two conditions. 
Clear SS-EPs were recorded in both conditions. Furthermore, the scalp topographies of the SS-EPs measured in the two conditions were highly similar, both being maximal over the left parietal regions, contralateral to the stimulated side, being compatible with activity predominantly originating from the primary somatosensory cortex.

In summary, the present study presents a novel method to compare, using EEG, the cortical processing of vibrotactile input during dynamic vs. passive tactile exploration of a textured surface. The obtained results suggest that the employed methodology can be used to explore the brain responses in the two conditions.

To our knowledge, this is the first study using SS-EPs to compare, in humans, the cortical activity in response to tactile input generated during voluntary and externally produced movements.

\section{ACKNOWLEDGMENTS}

The authors would like to thank Sébastien Wilmet for excellent technical assistance.

\section{REFERENCES}

[1] F. McGlone, E. F. Kelly, M. Trulsson, S. T. Francis, G Westling, and R. Bowtell, "Functional neuroimaging studies of human somatosensory cortex," Behav Brain Res, vol. 135, pp. 147-58, Sep 202002 .

[2] R. Kitada, T. Hashimoto, T. Kochiyama, T. Kito, T. Okada, M. Matsumura, et al., "Tactile estimation of the roughness of gratings yields a graded response in the human brain: an fMRI study," Neuroimage, vol. 25, pp. 90-100, Mar 2005.

[3] T. Harada, D. N. Saito, K. Kashikura, T. Sato, Y. Yonekura, M. Honda, et al., "Asymmetrical neural substrates of tactile discrimination in humans: a functional magnetic resonance imaging study," J Neurosci, vol. 24, pp. 7524-30, Aug 252004.

[4] J. J. Gibson, "Observations on active touch," Psychol Rev, vol. 69, pp. 477-91, Nov 1962

[5] C. E. Chapman, "Active versus passive touch: factors influencing the transmission of somatosensory signals to primary somatosensory cortex," Can J Physiol Pharmacol, vol. 72, pp. 558-70, May 1994.

[6] S. Lederman, "The perception of surface roughness by active and passive touch," Bulletin of the Psychonomic Society, vol. 18 pp. $253-255,1981$

[7] M. Hollins and S. R. Risner, "Evidence for the duplex theory of tactile texture perception," Percept Psychophys, vol. 62, pp. 695705, May 2000.

[8] M. A. Srinivasan, J. M. Whitehouse, and R. H. LaMotte, "Tactile detection of slip: surface microgeometry and peripheral neural codes," J Neurophysiol, vol. 63, pp. 1323-32, Jun 1990.

[9] J. R. Phillips, K. O. Johnson, and H. M. Browne, "A comparison of visual and two modes of tactual letter resolution," Percept Psychophys, vol. 34, pp. 243-9, Sep 1983.

[10] J. W. Morley, A. W. Goodwin, and I. Darian-Smith, "Tactile discrimination of gratings," Exp Brain Res, vol. 49, pp. 291-9, 1983.

[11] M. A. Heller, "Active and passive tactile braille recognition," Bulletin of the Psychonomic Society, vol. 24, pp. 201-202, 1986.

[12] V. Cronin, "Active and passive touch at four age levels," Developmental Psychology, vol. 13, pp. 253-256, 1977.

[13] G. Cheron, B. Dan, and S. Borenstein, "Sensory and motor interfering influences on somatosensory evoked potentials," $J$ Clin Neurophysiol, vol. 17, pp. 280-94, May 2000.
[14] C. E. Chapman, M. C. Bushnell, D. Miron, G. H. Duncan, and J. P. Lund, "Sensory perception during movement in man," Exp Brain Res, vol. 68, pp. 516-24, 1987.

[15] C. E. Chapman, W. Jiang, and Y. Lamarre, "Modulation of lemniscal input during conditioned arm movements in the monkey," Exp Brain Res, vol. 72, pp. 316-34, 1988.

[16] D. F. Collins, T. Cameron, D. M. Gillard, and A. Prochazka, "Muscular sense is attenuated when humans move," $J$ Physiol, vol. 508 ( Pt 2), pp. 635-43, Apr 151998.

[17] C. Ghez and G. L. Lenzi, "Modulation of sensory transmission in cat lemniscal system during voluntary movement," Pflugers Arch, vol. 323, pp. 273-8, 1971.

[18] K. Seki, S. I. Perlmutter, and E. E. Fetz, "Sensory input to primate spinal cord is presynaptically inhibited during voluntary movement," Nat Neurosci, vol. 6, pp. 1309-16, Dec 2003.

[19] S. J. Blakemore, D. M. Wolpert, and C. D. Frith, "The cerebellum contributes to somatosensory cortical activity during self-produced tactile stimulation," Neuroimage, vol. 10, pp. 44859, Oct 1999.

[20] A. S. Scwharz, A. J. Perey, and A. Azulay, "A Further analysis of active and passive touch pattern discrimination," Bulletin of the Psychonomic Society, vol. 6, pp. 7-9, 1975.

[21] C. Simoes-Franklin, T. A. Whitaker, and F. N. Newell, "Active and passive touch differentially activate somatosensory cortex in texture perception," Hum Brain Mapp, vol. 32, pp. 1067-80, Jul 2011.

[22] M. C. Chang, S. H. Ahn, Y. W. Cho, S. M. Son, Y. H. Kwon, M. Y. Lee, et al., "The comparison of cortical activation patterns by active exercise, proprioceptive input, and touch stimulation in the human brain: a functional MRI study," NeuroRehabilitation, vol. 25, pp. 87-92, 2009.

[23] D. Regan, Human brain electrophysiology: Evoked potentials and evoked magnetic fields in science and medicine. New York: Elsevier, 1989.

[24] S. Tobimatsu, Y. M. Zhang, and M. Kato, "Steady-state vibration somatosensory evoked potentials: physiological characteristics and tuning function," Clin Neurophysiol, vol. 110, pp. 1953-8, Nov 1999.

[25] A. Z. Snyder, "Steady-state vibration evoked potentials: descriptions of technique and characterization of responses," Electroencephalogr Clin Neurophysiol, vol. 84, pp. 257-68, May-Jun 1992.

[26] C. M. Giabbiconi, N. J. Trujillo-Barreto, T. Gruber, and M. M. Muller, "Sustained spatial attention to vibration is mediated in primary somatosensory cortex," Neuroimage, vol. 35, pp. 25562, Mar 2007.

[27] E. Colon, S. Nozaradan, V. Legrain, and A. Mouraux, "Steadystate evoked potentials to tag specific components of nociceptive cortical processing," Neuroimage, vol. 60, pp. 571-81, Mar 2012.

[28] D. Kourtis, E. Seiss, and P. Praamstra, "Movement-related changes in cortical excitability: a steady-state SEP approach," Brain Res, vol. 1244, pp. 113-20, Dec 92008.

[29] E. Mallinckrodt, A. L. Hughes, and W. Sleator, Jr., "Perception by the skin of electrically induced vibrations," Science, vol. 118, pp. 277-8, Sep 41953.

[30] E. Vezzoli, M. Amberg, F. Giraud, and B. Lemaire-Semail, "Electrovibration Modeling Analysis," in Haptics: Neuroscience, Devices, Modeling, and Applications, Springer Berlin Heidelberg, 2014, pp. 369-376.

[31] T. Sednaoui, E. Vezzoli, B. M. Dzidek, B. Lemaire-Semail, C. Chiappaz, and M. Adams, "Experimental evaluation of friction reduction in ultrasonic devices," in World Haptics Conference, 2015.

[32] M. Biet, F. Giraud, and B. Lemaire-Semail, "Squeeze film effect for the design of an ultrasonic tactile plate," IEEE Trans. Ultrason. Ferroelectr. Freq. Control, vol. 54, pp. 2678-2688, 2007.

[33] T. Watanabe and S. Fukui, "A method for controlling tactile sensation of surface roughness using ultrasonic vibration," in IEEE International Conference on Robotics and Automation, 1995, pp. 1134-1139.

[34] M. Amberg, F. Giraud, B. Lemaire-Semail, P. Olivo, G. Casiez, and N. Roussel, "STIMTAC: A Tactile Input Device with 
Programmable Friction," in 24th Annual ACM Symposium Adjunct on User Interface Software and Technology, New York, 2011, pp. 7-8.

[35] M. Biet, G. Casiez, F. Giraud, and B. Lemaire-Semail, "Discrimination of Virtual Square Gratings by Dynamic Touch on Friction Based Tactile Displays," in Symposium on Haptic interfaces for virtual environment and teleoperator systems, 2008, pp. 41-48.

[36] A. Mouraux and G. D. Iannetti, "Across-trial averaging of eventrelated EEG responses and beyond," Magn Reson Imaging, vol. 26, pp. 1041-54, Sep 2008.

[37] A. Hyvarinen and E. Oja, "Independent component analysis: algorithms and applications," Neural Netw, vol. 13, pp. 411-30, May-Jun 2000.

[38] M. a. J. Frigo, S. G., "FFTW: An adaptive software architecture for the FFT. ," presented at the International Conference of Acoustics, Speech, and Signal Processing., Seattle, WA., 1998.

[39] S. Nozaradan, I. Peretz, M. Missal, and A. Mouraux, "Tagging the neuronal entrainment to beat and meter," J Neurosci, vol. 31, pp. 10234-40, Jul 132011.

[40] A. Mouraux, G. D. Iannetti, E. Colon, S. Nozaradan, V. Legrain, and L. Plaghki, "Nociceptive steady-state evoked potentials elicited by rapid periodic thermal stimulation of cutaneous nociceptors," J Neurosci, vol. 31, pp. 6079-87, Apr 202011.

[41] E. Porcu, C. Keitel, and M. M. Muller, "Concurrent visual and tactile steady-state evoked potentials index allocation of intermodal attention: a frequency-tagging study," Neurosci Lett, vol. 556, pp. 113-7, Nov 272013. 